\title{
De ambientes de aprendizaje hacia ambientes de desafío. Estado del arte en torno a los ambientes de clase
}

\section{From learning environments to challenging environments. State of art around class environments}

Recepción: 3 de mayo de 2015

Aceptación: 20 de noviembre de 2015

Artículo de Reflexión

\section{Resumen}

Este artículo da cuenta de los avances de un proyecto de investigación, titulado "Ambientes de Desafío, una estrategia de aprendizaje en el aula". Relaciona los trabajos que han desarrollado algunos investigadores, con respecto a los ambientes de aprendizaje y las estrategias que favorecen el reconocimiento de los sujetos que actúan en el acontecer del aula. Inicialmente, se abordan elementos del problema, relacionados con los modos de apropiación del conocimiento por parte de los estudiantes y las relaciones interpersonales que se establecen con sus maestros. Posteriormente, la revisión
Yeiny Rosmira Gutiérrez Pinto* Martha Yaneth Perilla Rojas**

documental, desde los ambientes de aprendizaje, permitió hacervisibles algunas categorías emergentes, como los aspectos socio-emocionales y la denominación de desafíos, que dotaron de nuevos sentidos y significados a la propuesta. Finalmente, se muestran vacíos encontrados y se establecen puntos de llegada, con miras a la transformación de la práctica docente, en la Institución Educativa Técnica José Ignacio de Márquez del municipio de Ramiriquí.

Palabras clave: estado del arte, ambientes de aprendizaje, desarrollo socio-emocional, ambientes de desafío, ritmos de aprendizaje.
*Institución Educativa Técnica José Ignacio de Márquez - Boyacá Colombia yeinygutierrezpinto@ botmail.com

**institución Educativa Técnica José Ignacio de Márquez - Boyacá Colombia mayapero2@gmail.com

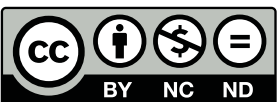




\section{Abstract}

This article reports on the progress of a research project, entitled "Environments of Challenge, a strategy of learning in the classroom". It relates the work that some researchers have developed, with respect to the learning environments and the strategies that favour the recognition of the subjects that act in the happening of the classroom. Initially, elements of the problem are addressed, related to the ways in which students acquire knowledge and the interpersonal relationships established with their teachers. Subsequently, the documentary review, from the learning environments, allowed making visible some emerging categories, such as socioemotional aspects and the naming of challenges, which gave new meanings to the proposal. Finally, gaps are found and arrival points are established, pointing to the transformation of teaching practice, in "José Ignacio de Marquez" Technical Educational Institution in Ramiriquí (Boyacá, Colombia).

Keywords: state of the art, learning environments, socio-emotional development, challenging environment, learning rhythms 


\section{Introducción}

Este artículo da cuenta de los avances de un proyecto de investigación, en torno de los ambientes de aprendizaje, y su contraste con diferentes autores y teorías, a partir de una revisión de estado del arte.

$\mathrm{Su}$ importancia radica en reconocer a la escuela, y específicamente al aula, no solamente como un lugar de negociación de saberes, construcción de conocimientos, circulación de metodologías; sino también, en un escenario de relaciones, de interacción entre los sujetos, propicio para la configuración de las subjetividades humanas (Freire, 1997).

A partir de la mirada de las autoras, se crean ambientes que pueden o no dinamizar aprendizajes y actitudes de quienes habitan elaulay son co-partícipes en la actividad educativa. De igual modo, se establecen ciertas relaciones, a veces verticalistas, en donde es el maestro quien se atribuye el dominio sobre los estudiantes, quienes, a su vez, asumen actitudes pasivas y de obediencia. No obstante, se pueden crear ambientes de participación activa, reconocedores de la VOz y de las potencialidades de los sujetos aprendientes, en ambientes que replantean el rol docente, en contextos de aprendizaje caracterizados por lógicas en la clase más horizontales, donde la construcción de conocimiento $\mathrm{y}$ de relaciones intersubjetivas, se hacen más democráticas.

Desde esta perspectiva, las autoras son conscientes de que las prácticas docentes y el ambiente del aula son susceptibles de ser modificadas, en el contexto de una realidad educativa en movimiento; y de la necesidad de reconocer al sujeto como parte vital en su aprendizaje. A estas ideas se ajustan los planteamientos de algunos autores, como Brophy y Wentzel (citados en Santrock, 2014), quienes dan importancia a la necesaria compatibilidad que debe existir entre lo que planea el maestro para la hora académica y la atención a las particularidades con que cuenta el grupo de estudiantes, apoyando de esta manera la iniciativa y fortaleciendo la autonomía de los educandos. Es importante señalar que, las contribuciones de los autores permitieron vislumbrar el camino a seguir en la elaboración de una estrategia novedosa en el contexto del aula, que permita atender a los vacíos encontrados durante el ejercicio de revisión documental.

En el presente estado del arte, se contextualiza el concepto 'ambiente de aprendizaje'. Con el fin de dar a conocer los significados e interpretaciones dadas desde diferentes autores, es preciso recalcar, en este aspecto, que dicho criterio permitió guiar la búsqueda de la información, haciendo visibles dos categorías emergentes que contribuyeron a definir el rumbo de la investigación, y que hicieron un aporte significativo a la formulación de una estrategia de aprendizaje para los grados 8-3 y 10-3 de la Institución Educativa mencionada, el cual es el objetivo principal del trabajo que se viene desarrollando.

Se hizo un ejercicio de revisión bibliográfica en diferentes fuentes, como tesis, libros y artículos científicos,
Este artículo da cuenta de los avances de un proyecto de investigación, en torno de los ambientes de aprendizaje, y su contraste con diferentes autores $y$ teorías, a partir de una revisión de estado del arte. 
"Establecimiento de necesidades, probabilidades defuncionamiento, enfoque pedagógico, experiencias similares que demuestren frabilidad del ambiente $y$ delimitar la información encontrada para sustentar el éxito del ambiente" los cuales se constituyeron en aportes importantes para el trabajo realizado. Al respecto, cabe señalar, que uno de los autores que se aproximó al interés de la investigación, en relación con el tema propuesto, fue Santrock (citado en Moreno, 2010), quien utiliza el concepto de "atmosferas de desafío" (p. 33) para referirse a los ambientes de aprendizaje; concepto que permitió orientar la búsqueda en la revisión documental.

Es preciso aclarar que, en la literatura revisada, son muy pocas las investigaciones que se refieren al concepto 'ambientes de desafío' en lo que respecta al ámbito educativo; sin embargo, se asocia con un concepto más conocido como es "Ambientes de aprendizaje", noción que dio la posibilidad de encausar el estado del arte.

En este orden de ideas, y luego del análisis de treinta fichas de revisión bibliográfica, se consolidan las categorías de ambientes de aprendizaje; entendido como el conjunto de condiciones necesarias para la comprensión del conocimiento y la motivación en el contexto del aula. De igual manera, el desarrollo socio-emocional que atiende los aspectos ligados al querer y sentir de los sujetos de la escuela; y los desafíos, categoría entendida como la sinergia entre múltiples actividades, que se constituyen en retadoras para el desarrollo de habilidades en los estudiantes, a partir de las posibilidades que le brinda el maestro.

Es propósito de las autoras, que el artículo suscite la creación y recreación de estrategias que motiven y desafíen el aprendizaje en el aula y que convoque la reflexión de la propia práctica, a partir de este ejercicio de revisión teórica.

\section{Aspectos teóricos}

A continuación, se realizará una descripción del concepto ambientes de aprendizaje que orientó el estado del arte y, a su vez, la estrategia de intervención propuesta en el proyecto de investigación.

\subsection{Ambientes de aprendizaje}

Tomando el concepto aportado por el Ministerio de Educación Nacional MEN (s.f.),

Un ambiente de aprendizaje, es un espacio en el que los estudiantes interactúan, bajo condiciones y circunstancias físicas, humanas, sociales y culturales propicias, para generar experiencias de aprendizaje significativo y con sentido. Dichas experiencias son el resultado de actividades y dinámicas propuestas, acompañadas y orientadas por un docente (p. 1).

Las características generales con que cuenta un ambiente de aprendizaje, implican condiciones y circunstancias que implementen estrategias pedagógicas, que, a su vez, dinamicen las actividades de enseñanza y rompan con la rutina y la fragmentación del conocimiento. Para construir ambientes de aprendizaje, según Rodríguez (2013), es importante atender a criterios como: "Establecimiento de necesidades, probabilidades de funcionamiento, enfoque pedagógico, experiencias similares que demuestren fiabilidad del ambiente y delimitar la información encontrada para sustentar el éxito del ambiente" (p. 5). 
Ahora bien, en el documento orientador del foro educativo nacional 2014, se plantea que:

Las propuestas de política pública actuales ponen el énfasis en que el sistema educativo garantice a todos los niños, niñas y jóvenes una educación de la misma calidad para todos, para lo cual debe ofrecer oportunidades legítimas de progreso e inclusión. Se entiende que la educación de calidad, debe posibilitar que todos los estudiantes, sin distingo de raza, género, lengua, religión, etnia, cultura, condición económica, social y/o física; aprendan en ambientes y entornos escolares que reconozcan las potencialidades individuales y las desarrollen (MEN, 2014, p. 6).

Desde la postura de esta institución del estado, se hace necesario que dichos ambientes trasciendan el aula de clase, llegando a atender, de manera consciente y detallada, las particularidades que se presentan en los contextos de cada institución educativa; permitiéndoles a los estudiantes, sentirse parte importante de la planeación que el maestro hace para aproximarlos al conocimiento.

A parte de eso, el Proyecto Educativo Institucional (PEI) de la Institución Educativa Técnica José Ignacio de Márquez del municipio de Ramiriquí, expresa en la misión y gestión estratégica, su aspiración como institución por responder a la diversidad, procurando ambientes agradables; así mismo, los objetivos institucionales y los fundamentos pedagógicos, sociales y axiológicos, reafirman la necesidad de ambientes de aprendizaje agradables en el entorno escolar, que desarrollen las competencias ciudadanas, la democracia, la autonomía y el sentido crítico en los educandos (PEI, 2015).

De acuerdo con lo anterior, las autoras le apuestan a la resignificación de las prácticas docentes que permitan gestar ambientes de aprendizaje, potencializadores de las competencias propuestas en el derrotero institucional.

\section{Estado del arte}

Como punto de partida, se citan las ideas de Londoño, Maldonado y Calderón (2014), quienes consideran que el estado del arte es una etapa de la investigación que permite revisar el estado del conocimiento acerca de un tema, y determinar cuáles son las tendencias. De esta manera, este ejercicio de síntesis sirvió como referencia para asumir una postura crítica frente a lo que se ha hecho o falta por hacer en cuanto a los ambientes de aprendizaje. A continuación, se relacionan las categorías:

\subsection{Ambientes de aprendizaje}

En este apartado, se logra visibilizar tres subcategorías que se constituyen como necesarias en los ambientes de aprendizaje, las condiciones humanas, socio-culturales y físicas que hacen parte del aula.

2.1.1 Condiciones humanas. Entre los autores que involucran al estudiante como parte importante del ambiente de aprendizaje, están: Medina (1980) quien indica que, para promover la autonomía en el educando, se requiere contribuir a la generación de un clima
Como punto de partida, se citan las ideas de Londoño, Maldonado y Calderón (2014), quienes consideran que el estado del arte es una etapa de la investigación que permite revisar el estado del conocimiento acerca de un tema, y determinar cuáles son las tendencias. 
de aula cooperativo y empático. A su vez, Alvarado (2015) plantea que "los ambientes de aprendizaje centrados en el estudiante, fomentan la responsabilidad y la colaboración, promueven el estudio e investigación en contexto e impulsan actividades de participación activa, y procesos de pensamiento de alto nivel" (p. 3).

Por su parte, López (2009) en su artículo científico, que es una compilación de diferentes estudios e investigaciones, demuestra que la motivación es de vital importancia para el educando, dado que genera el interés por el aprendizaje y se constituye en motor para que el estudiante asista al salón de clase por gusto, aunque en ocasiones asista a la escuela por obligación. Ahora bien, esto se logra si el estudiante puede ponerse a prueba a sí mismo, por lo cual se debe permitir que el educando tenga poder de decisión en los procesos de adquisición del conocimiento.

Lo anterior da cuenta del lugar significativo que conceden los autores mencionados, a los estudiantes $\mathrm{y}$ maestros como actores centrales en la generación de mejores condiciones para los ambientes de aprendizaje. En

López (2009) en su artículo cientifico, que es una compilación de diferentes estudios $e$ investigaciones, demuestra que la motivación es de vital importancia para el educando, dado que genera el interés por el aprendizaje y se constituye en motor para que el estudiante asista al salón de clase por gusto, aunque en ocasiones asista a la escuela por obligación. tal sentido, otros aspectos, como la responsabilidad y la colaboración, se aúnan a dichas condiciones, asumidos como mecanismos que activan la participación del estudiante en las actividades del aula, propiciando un escenario dialógico y empático en virtud del aprendizaje.

De igual manera, muchos autores convocan a los sujetos docentes en la generación de ambientes de aprendizaje propicios para el mejoramiento de la calidad educativa, entre ellos Lira y Vela (2013) quienes indican que los maestros deben conocer más que la materia que imparten, puesto que una de las habilidades necesarias, es conocer a fondo a sus estudiantes, lo que les permitirá atender adecuadamente a sus intereses. En esta misma línea, Uldemolíns \& de la Rubia-Valladolid (2013) indican que "Hay que romper el tópico del profesor de Matemáticas que vive en su mundo científico, despreocupado de la Didáctica, y cuyas explicaciones son inalcanzables para la mayoría de los mortales" (p. 3). Más aún, Jaramillo y Ruiz (2012) expresan:

Una manera efectiva que permitiría al docente competir eficazmente con los distractores en el aula es implementando diversas estrategias metodológicas y material didáctico que resulten atractivos para el estudiante, capturando su atención y motivando su participación en el desarrollo de las actividades propias de la clase (p. 6).

Así pues, según Balbuena (2009), "Si modificamos los contenidos pero mantenemos los métodos, el resultado, posiblemente, será que todo sigue igual" (p. 4). Por tanto, la labor docente en la generación de ambientes propicios de aprendizaje, reclama la necesidad de hacer ruptura de la monotonía de las clases, y esto se logra cuando docentes y estudiantes se ven constantemente en desequilibrios cognitivos que generen reto, emoción y deseos de superación. En relación con las ideas anteriores, las autoras señalan, además, la necesidad de desarrollar habilidades en los estudiantes, que les permita reconocer sus potencialidades para el aprendizaje. 
De otro lado, y a partir de lo que señalan los autores mencionados, llama la atención a los maestros, a estar alerta frente al encapsulamiento disciplinar, refiriéndose a sus discursos y actuaciones en el aula, pues pudieran opacar la intervención de los alumnos, en la creación de un ambiente de aprendizaje en donde todos los actores sean sujetos propositivos y participativos. En este sentido, mientras no se modifiquen los métodos utilizados por el maestro, difícilmente habrá transformación del ambiente de aula, que convoque un aprendizaje comprensivo por parte de los estudiantes.

\subsubsection{Condiciones sociales y} culturales. Cabe resaltar los aportes de Gromero (s.f.) quien revela que el interés de un estudiante depende del contexto social en que se desarrolla y de los contenidos básicos que aprende; sin embargo, su argumentación desatiende a la necesidad de vincular además la cultura arraigada en las sociedades presentes, donde el trabajo intelectual se ha quedado para unos pocos, en atención a que en la mayoría de los hogares no se enfatiza la necesidad de la superación personal y el fortalecimiento de los proyectos de vida. Es significativo el aporte relacionado con contenidos básicos que hace el autor, interpretándose como los aspectos fundamentales que necesita saber un estudiante para comprender conceptos posteriores.

De igual manera, Parra et al. (1994) indican que es necesario convertir la hora académica en un momento grato, rompiendo con los círculos viciosos de rituales ajenos a los intereses de los alumnos, ya que esto beneficiará la función que debe cumplir la escuela; se une a lo expresado por Assmann (2002) quien afirma que "El ambiente pedagógico tiene que ser un lugar de fascinación e inventiva, no inhibir sino propiciar la dosis de ilusión común entusiasta requerida para que el proceso de aprender se produzca como mezcla de todos los sentidos" (p. 28). Se reconoce que los rituales ajenos a los intereses de los estudiantes, pueden referirse a todas aquellas prácticas institucionalizadas por el maestro y los sistemas educativos, las cuales se repiten una y otra vez, prácticas que pudieran causar un efecto contrario, reflejado en la desmotivación de los educandos hacia el aprendizaje. Se considera que una estrategia apropiada, necesariamente debe aportar y mover la motivación de los alumnos, haciéndolos parte vital de la clase, permitiéndoles decidir, y vinculándolos en diferentes actividades que le ayuden a fortalecer su autoestima y confianza. Cabe destacar la denominación que le da Assmann a los ambientes de aprendizaje, como ambiente pedagógico, apuntando el concepto más hacia las prácticas de enseñanza que hacia las de aprendizaje, y reconociendo que la tan mencionada ilusión común entusiasta difícilmente se dará, entre tanto el maestro quiera homogeneizar al grupo y no permita los agrupamientos flexibles, para que todo el mundo aprenda según su ritmo.

Ahora bien, Vázquez, Jiménez, Mellado y Toboada (2007) argumentan que los procesos de innovación no pueden ser entendidos como la aplicación de nuevas teorías o prácticas que rivalicen $\mathrm{O}$ intenten contraponerse a viejos esquemas. Por lo tanto, la innovación trasciende las teorías y va más allá, procurando que la mayoría de
Cabe resaltar los aportes de Gromero (s.f.) quien revela que el interés de un estudiante depende del contexto social en que se desarrolla y de los contenidos básicos que aprende; sin embargo, su argumentación desatiende a la necesidad de vincular además la cultura arraigada en las sociedades presentes, donde el trabajo intelectual se ba quedado para unos pocos, en atención a que en la mayoría de los bogares no se enfatiza la necesidad de la superación personal y el fortalecimiento de los proyectos de vida. 
En esta misma línea, Galindo y Suárez (2011) argumentan que en los centros escolares, los docentes no cuentan con estrategias novedosas, utilizan poco los recursos y dedican más tiempo a actividades de repetición, lo cual dificulta el aprendizaje. los discentes, se sienten vinculados e interesados por la clase, porque saben que es allí donde hay un lugar para ellos, escogido por ellos, donde se valora su desempeño así sea de forma básica. Por lo tanto, llevando los aportes de estos autores a la cotidianidad del aula, se podría decir que la clase magistral no es del todo mala, lo que necesita es permitir mayores cuestionamientos y diversificar sus modos de valoración, con miras a generar autonomía, participación, y procesos de transición hacia ambientes más constructivistas.

2.1.3 Condiciones físicas. $\mathrm{Al}$ referirse a este contexto, se convoca a Augustowksy (2004) quien considera que, para la enseñanza, «Habitar significa apropiarse reflexiva y emocionalmente de los espacios y convertir los escenarios que nos presentan las instituciones en "buenos" y ¿por qué no? - "bellos" lugares de trabajo, en sitios adecuados para enseñar y para aprenden» (p. 16). Todavía cabe considerar que, la adecuación de estos lugares debe ser un trabajo conjunto y concertado entre los sujetos vivos que utilizan el espacio del aula de clase, permitiendo al estudiante personalizar su lugar de interacción con el conocimiento. Cabría aquí también preguntarse, si realmente las aulas de clase constituidas como hasta el momento se han conocido, podrían llamarse como lo indica el autor, sitios adecuados para enseñar y aprender. Se considera en este punto que, dichos sitios adecuados necesitan valerse de todos y cada uno de los elementos que ofrece el entorno, para resignificar los conceptos abstractos de las diferentes asignaturas.

Más aún, Osorio y Duarte (2011) indican que las actividades auténticas requieren hacer mejor aprovechamiento de los ambientes, fundando condiciones para un mayor aprendizaje y mejores resultados académicos. En esta misma línea, Galindo y Suárez (2011) argumentan que en los centros escolares, los docentes no cuentan con estrategias novedosas, utilizan poco los recursos y dedican más tiempo a actividades de repetición, lo cual dificulta el aprendizaje. Interesa de los conceptos aportados por estos autores, las denominadas actividades auténticas, que podrían expresarse como actividades retadoras, las cuales aprovechen los recursos que el contexto pueda ofrecerles, con miras a lograr desafiar a los estudiantes, sacándolos de sus posiciones pasivas y obedientes, adoptadas ante las interacciones verticalistas del docente, que afectan directamente el compromiso que los educandos puedan asumir con su preparación, el sentido de pertenencia con su institución y su deseo de persistir en su preparación intelectual luego de abandonar el colegio. En contraste, las autoras del presente artículo, consideran que, no solo interviene el ambiente, sino, a su vez, el aprovechamiento de las potencialidades de los sujetos estudiantes y sujetos docentes.

\subsection{Aspectos socio-emocionales}

En relación con esta categoría, los autores reconocen que, cuando el maestro potencia la capacidad cognitiva, junto al desarrollo de habilidades socioemocionales, beneficia directamente la apropiación del conocimiento de manera positiva, entendiendo, como desarrollo socio-emocional a la correcta vinculación del estudiante con sus proyectos de futuro, reconociéndose 
como sujeto capaz de automotivarse y perseverar en su formación como personal integral.

$\mathrm{Al}$ respecto, cabe resaltar los aportes de Spranger (1960) quien enfatiza que el clima del amor dentro de la relación concreta educativa, es la única forma de llegar al centro personal del sujeto. Desde la opinión de las autoras, articuladas a esta idea, se encuentra que tanto la afectividad, como la atención a los intereses cognoscibles del educando por parte del maestro, son necesarias. Incluso, se relaciona ese centro personal del sujeto con el interés que pueda generarle las responsabilidades educativas, logrando movilizar así la motivación del estudiante.

Asimismo, Mager (1971) indica que "Cualquier cosa que hagamos con miras a influir sobre el educando, lo menos que debemos esforzarnos en conseguir, es que se aleje de nosotros con sentimientos favorables" (p. 24). Igualmente, parafraseando a González (1995), es fundamental el ejemplo del profesor y las relaciones positivas con este, generadas más por respeto y confianza, que por imposición o miedo. Adicionalmente, Anaya, Ibaven, González y Maldonado (s.f.) concluyeron en su investigación, que los alumnos de secundaria concedieron más peso a la relación interpersonal con sus maestros, que a aspectos meramente formales del habla. Por lo tanto, los estudiantes dan cuenta del lugar privilegiado que ocupan las relaciones interpersonales en medio de un ambiente de aula, sobre los contenidos manejados por el maestro, sobre todo cuando son utilizados con un lenguaje técnico que dificulta su comprensión.
De la misma manera, Gómez, Mir y Serrats (1995) argumentan que el maestro debe favorecer el equilibrio afectivo, evitando el desánimo y el estrés en los estudiantes. Equilibro afectivo se entiende como la correcta atención al desarrollo socio-emocional del estudiante. Una propuesta que atienda a los argumentos de los autores, debe lograr discentes responsables, comprometidos, emocionados, laboriosos, disciplinados, mucho más autónomos, con iniciativa, y aportando a su formación de acuerdo con sus posibilidades.

Al mismo tiempo, es importante enfatizar los resultados del estudio de: Wang, Haertel y Walberg (citados en Berger et al., 2009) quienes mencionan: "Se estimó la influencia relativa de 30 categorías diferentes sobre el aprendizaje de variables educacionales, psicológicas y sociales, demostrándose que las variables emocionales y sociales ejercían la mayor influencia en el desempeño académico" (p. 3). Igualmente, Extremera y FernándezBerrocal (citados en Berger et al., 2009) argumentan que: "El desarrollo socio emocional ha sido un área subinvestigada, muchas veces quedando en segundo plano dado el énfasis otorgado al desarrollo cognitivo" (p. 4). Hay que mencionar, además, que las relaciones intersubjetivas entre maestros y estudiantes, y el clima de aula, son generadoras del aprendizaje mutuo. En tal sentido, la potenciación de esta dimensión humana permite crear efectos vinculantes entre los sujetos vivos del aula. De este modo, los aportes de estos autores dan cuenta de dicha dimensión, como eje articulador intersubjetivo, necesario para un buen ambiente educativo, favorable al
Mager (1971) indica que "Cualquier cosa que hagamos con miras a influir sobre el educando, lo menos que debemos esforzarnos en conseguir, es que se aleje de nosotros con sentimientos favorables" 
De la Rosa (2011) revela que la actitud necesita de un componente afectivo desarrollado por la emoción ante la presencia real de un objeto de aprendizaje, $y$ la cual puede ser positiva o negativa, dependiendo del acercamiento asertivo hacia el mismo. aprendizaje y rendimiento académico de los alumnos.

Por su parte, Bacete y Doménech (1997) señalan que divergen: "Mientras los efectos de las emociones positivas pueden ser beneficiosos en la mayoría de los casos, el impacto de las emociones negativas como insatisfacción $\mathrm{O}$ ansiedad pueden ocasionar efectos ambivalentes.” (p. 11). En tal sentido, el maestro debe convertirse en un sujeto dinamizador de las actitudes de los estudiantes y promotor de sentimientos favorables que estrechen relaciones intersubjetivas motoras, en un ambiente para el aprendizaje.

Ahora bien, Diez (2011) expresa que "Lo cognitivo y lo afectivo mantienen relaciones de mutua dependencia" ( $p$. 7). Dichos argumentos permiten dar cuenta de una relación recíproca entre los aspectos afectivos y cognitivos, dimensiones, que al ser reconocidas por el maestro en sus intencionalidades pedagógicas, constituyen elementos valiosos para la generación de ambientes propicios para el aprendizaje. En el correcto amarre que se haga de estos dos aspectos con las actividades propuestas por el maestro, se estará aportando significativamente al favorecimiento del interés del estudiante, movilizando su estancamiento intelectual.

De la Rosa (2011) revela que la actitud necesita de un componente afectivo desarrollado por la emoción ante la presencia real de un objeto de aprendizaje, y la cual puede ser positiva o negativa, dependiendo del acercamiento asertivo hacia el mismo. Por su parte, Mendiburu (2011) afirma que los proyectos de futuro deben provenir de prácticas educativas en contextos de igualdad, solidaridad y coherencia social, haciendo que el futuro de la especie humana sea más esperanzador. Teniendo en cuenta lo anterior, el docente debe conocer a sus estudiantes y procurar que las relaciones de cooperación y confianza circulen en el aula, hay que permitirse nuevas formas de trabajar, donde las relaciones poco democráticas, sean transformadas en posibilidades de responsabilidad, donde los roles no estén tan trazados y definidos, y donde se evolucione la mirada de alguien que enseña y alguien que aprende, a la visión del trabajo interactivo que favorezca la resignificación de las prácticas educativas desde el lugar de los actores que intervienen en ella.

No obstante, algunos otros autores divergen de las anteriores apreciaciones, tal es el caso de Soria (2012) quien considera que:

Las buenas relaciones existentes entre profesores y alumnos pueden influir en el esfuerzo y trabajo que los alumnos realizan en sus materias de estudio, aunque esto no siempre es determinante, al comprobarse que si bien suele aumentarse en estos casos la motivación y esfuerzo, muchos alumnos se muestran neutrales y responsables en sus estudios independientemente del profesor, o incluso creen que deben esforzarse más en el caso de tener malas relaciones con estos ( $\mathrm{p}$. 90).

De conformidad con lo anterior, llama la atención que, aun cuando las buenas relaciones en el aula influyen en el esfuerzo y trabajo de los estudiantes, 
esto no se puede generalizar, pues el autor da cuenta de la actitud neutral e independiente que pueden asumir los estudiantes en la dinámica de su aprendizaje.

En esta misma línea, Polaino (citado en Bacete y Doménech, 1997) afirman que:

Una ansiedad moderada en las matemáticas, no solo disminuye el rendimiento sino que puede facilitarlo. Por el contrario, un nivel muy alto de ansiedad inhibe notablemente el rendimiento, ya que aparece como un factor disruptivo de los procesos motivacionales y cognitivos que son los que intervienen directamente sobre las habilidades y destrezas necesarias para la solución de problemas (p. 11).

Este autor demuestra, con sus argumentos, armonía con Lira y Vela (2013), en lo relacionado con las actividades que fomentan ansiedad en los estudiantes. Lo anterior, en razón a que el maestro debe desarrollar habilidades para conocer quiénes son sus estudiantes y las lógicas que se dan en sus procesos individuales y colectivos para acceder al conocimiento.

\subsection{Desafíos}

Los autores que se relacionan a continuación, además de vincularse con los ambientes de aprendizaje y los aspectos socio-emocionales, son mucho más específicos en la denominación de desafíos, entendiéndolos como aquellas actividades que son pensadas, atendiendo los ritmos de aprendizaje y propendiendo por la vinculación del ser en el proceso de aprendizaje comprensivo. Entre estos autores, se encuentra Cordoba (s.f.) quien enfatiza que "Es necesario para fomentar la emancipación intelectual, promover la creación de conflictos pedagógicos que permitan asumir desafíos ante el aprendizaje y apropiación del conocimiento" (p. 7).

Se entiende entonces, que el autor se refiere a la emancipación intelectual, como un modo, en el que a los sujetos les es permitido pensar por sí mismos, como un acto de autonomía, en donde estos se atreven a tomar postura en relación con la dinámica del aula, sobre la forma como se ponen en juego diversos aspectos que son causantes de lo que el autor llama conflictos pedagógicos, y que posteriormente lo conducen a enfrentar los desafíos del aprendizaje con mayor iniciativa, pues se considera un sujeto capaz de interactuar.

De manera análoga, Freire (1997) considera que: "Lo que se debe hacer es enseñar cierto contenido, y desafiar al estudiante para que se vaya percibiendo, en y por su propia práctica, como sujeto capaz de saber" (p. 119). Desde este puntodevista, lapercepción decapacidad se verá fortalecida siempre y cuando el estudiante se vincule y persevere en el trabajo propuesto, ofreciéndole conocimientos suficientes, los cuales se identifican como convergentes con lo planteado por Gromero (s.f.) respecto de los contenidos básicos que aprende el estudiante, que le aporten herramientas para poder enfrentar, de manera exitosa, las labores propuestas por el maestro. Sin embargo, en el planteamiento que hace Freire, pareciera que dichos
Este autor demuestra, con sus argumentos, armonía con Lira y Vela (2013), en lo relacionado con las actividades que fomentan ansiedad en los estudiantes. 
Santrock(citado en Moreno, 2010) indica que para mejorar la motivación, es necesario crear atmósferas de desafío y de expectativas, proporcionando a los estudiantes confianza en sí mismos, para alcanzar los retos planteados, fortaleciendo la motivación intrinseca. desafíos son únicos y generalizados a todo el grupo de estudiantes, sin atender adecuadamente a las particularidades que están presentes en cada una de las aulas, es por esto que uno de los vacíos encontrados tiene que ver con la omisión que se da, en los aportes de la mayoría de los autores, respecto a la necesidad de fomentar la persistencia en el estudiante, logrando que busque la forma de sobrepasar las barreras de sus dificultades, buscando herramientas alternas al maestro, todo porque lo mueve su interés y su deseo de sobresalir de acuerdo con sus posibilidades.

Por otra parte, Santrock (citado en Moreno, 2010) indica que para mejorar la motivación, es necesario crear atmósferas de desafío y de expectativas, proporcionando a los estudiantes confianza en sí mismos, para alcanzar los retos planteados, fortaleciendo la motivación intrínseca. Se considera que el autor se refiere al término de atmósferas de desafío para aludir a los ambientes de aprendizaje, que trascienden las expectativas del estudiante y favorecen su autonomía, fortalecida, gracias a la planeación de la clase de forma mucho más heterogénea, donde hay flexibilidad en los niveles de dificultad exigidos al estudiante y donde esta flexibilidad también permea a la evaluación. Sin embargo, sería importante reconocer que, en dichas atmósferas de desafío, el trabajo se debe estructurar, de manera tal, que el progreso pueda ser evidenciado y monitoreado por el mismo estudiante.

Otros investigadores compilados en Santrock (2014), han logrado comprobar en sus indagaciones en escuelas norteamericanas, que el flujo ocurre cuando los individuos se involucran en desafíos que no les resultan demasiado difíciles ni demasiado fáciles. Entre estos investigadores, están: Shernoff (citado en Santrock, 2014) quien afirma que "Los estudiantes se comprometen más cuando participan en contextos que disfrutan y consideran desafiantes y relevantes" (p. 395). Se considera, a su vez, que dicho contexto se logra en la medida que genere expectativas, atendiendo a los niveles de dificultad que ofrece la clase y alejándose de la monotonía de actividades planeadas y pensadas por otros, en situaciones totalmente ajenas a los intereses de los alumnos. Ahora bien, Brophy (citado en Santrock, 2014) argumenta que:

Es más probable que ocurra flujo en áreas en que los estudiantes se siente desafiados y perciben que tiene un alto grado de habilidad; se sienten aburridos cuando sus habilidades son altas pero la actividad no supone un reto; sienten apatía si tanto la dificultad como los niveles de habilidad son bajos; y experimentan ansiedad cuando enfrentan una tarea difícil y creen que no tiene las habilidades adecuadas para realizarla (p. 395).

De acuerdo con los planteamientos de Brophy, cabría preguntarse ¿cómo crear un clima de aula, de tal forma que los educandos perciban un ambiente desafiante en el aprendizaje?, ¿qué otros factores hacen que el alumno tenga esta sensación, denominada por el autor como desafiante?, ¿qué los lleva a la desmotivación, rechazo o aburrimiento?, ¿este ambiente desafiante podría generar una nueva cultura educativa? Al intentar responder estas preguntas, se traen las ideas de 
Anderman y Anderman (citados en Santrock, 2014) quienes afirman que: "En lugar de sentirse amenazados por las tareas difíciles es común que los hagan sentirse desafiados y emocionados" (p. 400).

Se puede decir, desde la perspectiva de las autoras, que el estudiante puede sentirse desafiado en la medida que el docente haya estructurado las explicaciones iniciales, de manera que pueda proveerle conocimientos básicos para no frustrarse en el transcurso del trabajo con las diferentes temáticas. Sentirse desafiado se entiende como ese interés que subyace del individuo por afrontar la tarea, por cuanto esta ha sido creada especialmente para él y pensando en que, así sea de forma básica, él puede aportar en la construcción de su intelecto.

Los autores Bandura y Schunk (citados en Santrock, 2014) indican que: "Los investigadores han encontrado que la autoeficiencia y el logro mejoran cuando los estudiantes establecen metas que son específicas, próximas y que suponen un reto" (p. 403). Mientras que, Eccles (citado en Santrock, 2014) afirma que "Es mucho más probable que trabajen duro para alcanzar una meta si esperan tener éxito que si esperan fracasar" (p. 405). Se toma posición analizando los aportes, y reconociendo que los alumnos difícilmente podrán establecer metas de este tipo si el sistema, el maestro, la evaluación y las pruebas externas no se los permite, atendiendo que los direccionamientos educativos encasillan el pensamiento, sin permitir desarrollar las habilidades inherentes a cada ser humano. Importa resaltar aquí, que las exigencias universalizadas hacen que el estudiante, en muy poca medida, desarrolle las habilidades con que llega a la escuela. Los educandos, probablemente, desarrollan desmotivación por el sometimiento del que son objeto por parte del sistema educativo, con oportunidades hechas para unos pocos, a los que probablemente, les parezca también, que lo que les exige ese sistema, es nada, comparado con lo que ellos están en capacidad de dar. Una alternativa a esto, podría incluir niveles de dificultad que se aseguren de la apropiación de los conocimientos, atendiendo los ritmos de aprendizaje.

Wigfield et al. (citados en Santrock, 2014) establecen que hay que "conocer al niño lo suficiente para brindarle las cantidades correctas de desafío y de apoyo" (p. 410). Dichos autores se suman a Lira y Vela (2013) y Polaino (citado en Bacete y Doménech, 1997), tomando como aspecto común a las tres categorías generadas, el criterio de conocer a los estudiantes, esto, permitirá al maestro lograr grupos focales, que realicen jalonamientos más acordes a las necesidades de los educandos, con el apoyo de los que van a su ritmo, permitiendo que el andamiaje no se de en un solo grupo de trabajo, donde los más avanzados ayuden a los más rezagados; sino que, entre grupos flexibles, se genere este andamiaje que les permita atender a cada uno, a sus necesidades particulares de apropiación del conocimiento.

Por otro lado, Wentzel (citado en Santrock, 2014) manifiesta que: "La motivación de los estudiantes se optimiza, cuando los maestros les plantean tareas desafiantes en un ambiente orientado al material de aprendizaje significativo e interesante
Se puede decir, desde la perspectiva de las autoras, que el estudiante puede sentirse desafiado en la medida que el docente haya estructurado las explicaciones iniciales, de manera que pueda proveerle conocimientos básicos para no frustrarse en el transcurso del trabajo con las diferentes temáticas. 
Ames (1992) converge con Vygotsky (citado en Padilla, 2008), al indicar la importancia de un modelo de evaluación dinámica, que permita conocer las posibilidades o capacidades del alumno en el desarrollo de una tarea $o$ actividad. y apoyo suficiente a su autonomía e iniciativa" (p. 412). Cabe resaltar que, lo expresado por el autor apunta a despertar el interés de los alumnos hacia la clase y hacia la apropiación de los conocimientos, permitiendo a su vez al maestro trascender en sus prácticas educativas, resignificándolas y exigiéndose día a día creatividad e innovación, planeando actividades novedosas, para mantener a los estudiantes expectantes e interesados por la clase y monitoreando el desarrollo, progreso y mejoramiento continuo de los educandos, logrando así aportar de manera significativa a su formación.

Ames (citado en Bacete y Doménech, 1997) propone una serie de actuaciones instruccionales del profesor, encaminadas a favorecer las metas de aprendizaje en función de las dimensiones por él señaladas anteriormente. En relación con las tareas y actividades de aprendizaje, propone:

Seleccionar aquellas que ofrezcan retos y desafíos razonables por su novedad, variedad o diversidad. Respecto a la distribución de autoridad o responsabilidad, propone ayudar a los alumnos en la toma de decisiones, fomentar su responsabilidad e independencia y desarrollar habilidades de autocontrol. Por último, respecto a las prácticas de evaluación, las estrategias instruccionales más importantes que se deberían implementar son: centrarse sobre el progreso y mejora individual, reconocer el esfuerzo de los alumnos y trasmitir la visión de que los errores son parte del proceso de enseñanza y aprendizaje (p. 14).

Los aspectos relacionados con Bacete y Doménech (1997), se convierten en ángulos de lectura, articulándose con los intereses de las investigadoras, $y$ se han convertido en fuente de consulta, análisis y soporte teórico de la estrategia de aula que se piensa en la propuesta de intervención. Se replantean interacciones y se resignifican los conceptos de actividades de aprendizaje, autoridad, responsabilidad y evaluación, analizándolos desde otras perspectivas, y posibilita orientarlos hacia el favorecimiento de la vinculación del ser en su proceso de persistencia en la construcción de su intelecto.

Incluso, Ames (1992) converge con Vygotsky (citado en Padilla, 2008), al indicar la importancia de un modelo de evaluación dinámica, que permita conocer las posibilidades o capacidades del alumno en el desarrollo de una tarea o actividad. Haciendo lectura a este significativo concepto de evaluación dinámica, lo relacionamos como oportunidades de presentar al estudiante, más de una posibilidad a la hora de ser valorada su aprehensión de los conocimientos de manera comprensiva, dando la alternativa de optar, de poder decidir en todas las propuestas pedagógicas de las que el estudiante hace parte, porque el maestro le da esa posibilidad.

\section{Consideraciones finales}

Interesa resaltar, de lo afirmado por los diferentes autores, que las lógicas del aula se han visto permeadas por la mirada homogeneizante, tanto de los modelos educativos como de los docentes; quienes, en su afán por atender grupos numerosos, se olvidan de las particularidades de los alumnos y del reconocimiento de sus potencialidades, 
y a los docentes de poder centrar más su mirada en la forma de atender la complejidad del grupo, por medio de estrategias mucho más efectivas y heterogéneas. $\mathrm{Al}$ respecto, conviene decir que los desafíos generan una sinergia entre las actividades retadoras y las habilidades de los estudiantes; y al docente, le permiten encontrar un mayor sentido y significado a su práctica educativa, más allá de una mirada reproduccionista de contenidos como convencionalmente se ha venido desarrollando, además de atender de una mejor manera los ritmos de aprendizaje.

Se relacionan a continuación, algunos aportes a partir de la revisión del estado del arte, tal es el caso de las diferentes denominaciones que ha tomado el concepto de ambientes de aprendizaje, como lo es ambiente pedagógico, clima de clase y hora académica, los cuales, en general, reconocen las condiciones humanas, socio-culturales y físicas que median los procesos de enseñanzaaprendizaje; sin embargo, dentro de las perspectivas novedosas para abordar el objeto de estudio, se proyecta el término 'atmósferas de desafío' que, vinculado con la última categoría dentro del artículo, enrutaron a las investigadoras para vislumbrar una estrategia innovadora, que permita mostrar caminos a seguir para atender los vacíos encontrados, considerar la subjetividad humana, la idoneidad, y reconocer la $\mathrm{VOZ}$ del estudiante en interacciones más horizontales y democráticas; logrando así trascender del concepto de Ambientes de Aprendizaje al concepto de Ambientes de Desafío, que va más allá de las condiciones hasta hoy reconocidas en los procesos de apropiación del conocimiento.
Paralelamente, se relacionan otros conceptos relevantes en la construcción de ambientes de desafío. En primera instancia, se vincula el concepto de conflicto pedagógico y desequilibrios cognitivos, asumiéndolos como análogos, e identificándolos como aporte novedoso de los modos de apropiación del conocimiento por parte de los estudiantes, haciendo ruptura de la monotonía de la clase magistral, incentivando retos, emoción, deseos de superación, y reconocimiento de la vOZ y las destrezas del educando.

En segunda instancia, el concepto de Emancipación Intelectual, entendida como el desarrollo de la confianza en sí mismo y, por consiguiente, de la iniciativa que lo conduce a no desertar, a esforzarse, a asumir su preparación intelectual porque la clase y el maestro se los permite y porque, además, se siente sujeto capaz de interactuar, gracias al fortalecimiento de su autonomía.

Por otra parte, la consideración que hace el autor Uldemolíns (2013) respecto de las explicaciones inalcanzables para la mayoría de los mortales, que dan cuenta, primordialmente, en el desarrollo de las ciencias duras, de aquellos ejercicios complejos que se quiere que todos los discentes manejen a la perfección, homogeneizando las exigencias, sin secuencializar el conocimiento, con miras a mejorar la comprensión por parte de los estudiantes.

Luego del ejercicio teórico, vale la pena decir que, aunque se han realizado trabajos referentes a los ambientes de aprendizaje, también se identifica que en esta exploración no se visualizan estrategias específicas en el aula, que
Al respecto, conviene decir que los desafíos generan una sinergia entre las actividades retadoras y las habilidades de los estudiantes 
permitan el reconocimiento del sujeto en el proceso de enseñanza-aprendizaje. Dichas estrategias de intervención deben tener muy en cuenta que, los ambientes adecuados de clase se fundamenten en el reconocimiento de los discentes, como parte importante del quehacer escolar, y esto se logra cuando las prácticas educativas se enfocan en un continuo análisis con intenciones de mejoramiento, que propendan por lograr educandos más interesados, con posibilidades de decidir y hacer en la medida de sus capacidades.

Así mismo, todo esto reducirá el riesgo de que los docentes midan a los estudiantes con la "misma vara", sin atender a sus individualidades y diferencias; puesto que, solo se estarían reafirmando las barreras de comunicación, que durante años se legitimaron en la escuela. Con todo y esto, se debe lograr que los alumnos comprendan la importancia de su hacer en el aula y, sobre todo, mejorar las relaciones interpersonales con sus compañeros y maestros; de otro modo, ¿cómo se puede hablar de calidad en los procesos de enseñanza-aprendizaje?

En este orden de ideas, las autoras dan relevancia a la reflexión, dado que se constituye en un fundamento de transformación del quehacer del maestro, permitiendo replantear sus prácticas pedagógicas tradicionales, innovando sus estrategias y contribuyendo a reforzar otros comportamientos que permitan obtener la construcción de nuevos ambientes de aprendizaje de los cuales, serán sus estudiantes, ahora en su lugar de sujetos activos del aula, quienes darán cuenta.

\section{Referencias}

Alvarado, C. (2015). Ambientes de aprendizaje en Física: Evolución hacia ambientes constructivistas. Latin-American Journal of Physics Education is the property of Latin-American Physics Education Network, 9(1). Recuperado el 8 de diciembre de 2015, de http://www.lajpe.org/jul15/S1203_Alvarado_2015.pdf.

Ames, C. (1992). Classrooms: Goals, structure, and student motivation. Journal of Educational Psychology, 84, 261-271.

Anaya, A., Ibaven, M., González, B., \& Maldonado, M. (s.f.). El discurso en el aula. Competencia comunicativa del docente en secundaria. En el Primer congreso internacional de Educación, "Construyendo inéditos viables” (pp. 1155-1168). Universidad Autónoma de Chihuahua, Chihuahua, México. Recuperado el 25 de marzo de 2016, de http://cie.uach.mx/cd/docs/area_05/a5p1.pdf.

Assmann, H. (2002). "Reencantar" la educación. Placer y Ternura en la Educación hacia una sociedad aprendiente. Madrid, España: Narcea, S.A. De Ediciones.

Augustowksy, G. (2004). Las paredes del aula. Buenos Aires, Argentina: Amorrortu editores.

Bacete, F. G., \& Doménech, F. (1997). Motivación, aprendizaje y rendimiento escolar. Revista española de motivación y emoción, (1), 55-65. Recuperado el 14 de junio de 2016, de http://reme.uji.es/articulos/pa0001/texto.html.

Balbuena, L. (2009). Reflexiones de un docente. Unión: revista iberoamericana de educación matemática, (17), 7-16. Recuperado el 24 de marzo de 2016, de http:/ / www.fisem.org/www/union/revistas/2009/17/Union_017_005.pdf. 
Berger, C., Milicic, N.,Alcalay,L., Torretti,A.,Arab,M., \& Justiniano, B. (2009).Bienestar socio-emocional en contextos escolares: la percepción de estudiantes chilenos. EstudiossobreEducación, 17,21-43. Recuperadoel8 de diciembre2015, dehttp:/ / biblio.uptc.edu.co:2103/ehost $/$ pdfviewer $/$ pdfviewer?vid=39\&sid=260b17acf45f-4be7-bff4-7b2562a1571c\%40sessionmgr4003\&hid=4106.

Cordoba, V. (s.f.). De aprender-Evaluación-Interacción, P. A. Poder $=$ Bloqueo $=$ Emancipación. Una mirada a la relación pedagógica entre el saber y el poder en el aprendizaje de la matemática. Recuperado el 25 de marzo de 2016, de http:/ / www.dfpd.edu.uy/departamentos/cs_educacion_phf/documentos/2012/ ForoCD/Textos/Valentina_Cordoba.pdf.

De la Rosa, L. (2011). Problemáticas y alternativas en la enseñanza de la química en la educación media en la isla de San Andrés, Colombia. (Tesis de Maestría). Universidad Nacional de Colombia, Bogotá, Colombia. Recuperado el 8 de marzo de 2016, de http://www.bdigital.unal.edu.co/4943/1/LuisRam\%C3\% B3ndelaRosaRodr\%C3\%ADguez.2011.pdf.

Diez, T. (2011). El uso de Applets en las matemáticas de la enseñanza secundaria. (Tesis de maestría). Recuperado el 5 de marzo de 2016, de http:// repositorio. unican.es/xmlui/bitstream/handle/10902/1643/Tamara\%20Diez\%20Sainz. pdf? sequence $=1$ \&is Allowed $=\mathrm{y}$.

Freire, P. (1997). Pedagogía de la autonomía. México: Siglo veintiuno editores.

Galindo, S., \& Suárez, N. (2011). Estrategias comunicativas en la dinamización de la clase de matemáticas. (Tesis de Maestría). Universidad Pedagógica y Tecnológica de Colombia, Tunja, Colombia.

Gómez, M. T., Mir, V., \& Serrats, M. G. (1995). Propuestas de intervención en el aula: Técnicas para lograr un clima favorable en la clase. Madrid, España: Narcea, S. A. de Ediciones.

González, D. G. (1995). Teoría de la Motivación y práctica profesional. Playa, Ciudad de la Habana: Editorial Pueblo y Educación.

Gromero, P. (s.f.). Estudiar Ciencias Duras... ¿Por Qué No? Recuperado el 24 de marzo de 2016, de http://www.ib.edu.ar/becaib/bib2011/trabajos/ GomeroEstrella.pdf.

Institución Educativa Técnica José Ignacio de Márquez. (2015). Proyecto Educativo Institucional (PEI). Ramiriquí: Comunidad Educativa.

Jaramillo, F., \& Ruiz, J. (2012). Una estrategia pedagógica para motivar procesos de aprendizaje en estadística descriptiva de básica secundaria. Silogismo más que conceptos, 10(1), 1-22. Recuperado el 5 de marzo de 2016, de http://www.cide. edu.co/ojs/index.php/silogismo/article/view/47/38.

Lira, Y., \& Vela, H. (2013). Docencia Integral. México, D.F.: Trillas S.A. de C.V.

Londoño, O. L., Maldonado, L. F., \& Calderón, L. (2014). Guía para construir estados del arte. Recuperado el 9 de diciembre de 2015, de http:/ / www.colombiaaprende. edu.co/ html/investigadores/1609/articles-322806_recurso_1.pdf.

López,E. (2009).Evaluacióndelefectodevariablescríticasenelaprendizajedelos escolares, EstudiossobreEducación, 16,55-78Recuperadoel8dediciembrede2015, dehttp:/ / biblio.uptc.edu.co:2103/ehost/pdfviewer/pdfviewer?vid=41\&sid=260b17acf45f-4be7-bff4-7b2562a1571c\%40sessionmgr4003\&hid=4106. 
Mager, R. (1971). Actitudes Positivas en la Enseñanza. México / Buenos Aires: Editorial Pax-México.

Medina, A. (1980). La Didáctica y la interacción en el aula. Bogotá, Colombia: Cincel S.A.

Mendiburu, I. V. (2011). Lev S. Vigotsky: la psicología cultural y la construcción de la persona desde la educación. En J. Trilla (Ed.), El legado pedagógico del siglo XX para la escuela del siglo XXI (pp. 207-227). Barcelona, España: Editorial Graó.

Ministerio de Educación Nacional. (2014). Documento orientador foro educativo nacional 2014: ciudadanos matemáticamente competentes. Recuperado el 10 de abril de 2017, de http:/ /www.colombiaaprende.edu.co/html/micrositios/1752/ articles-342931_recurso_1.pdf.

Ministerio de Educación Nacional. (s.f.). Portal Colombia aprende. Recuperado el 10 de abril de 2017, de http://www.colombiaaprende.edu.co/html/ productos/1685/w3-article-288989.html.

Moreno, A. (2010). Motivación y Desarrollo del pensamiento matemático. (Tesis de Maestría). Universidad Pedagógica y Tecnológica de Colombia, Tunja, Colombia.

Osorio, L., \& Duart, J. (2011). Análisis de la interacción en ambientes híbridos de aprendizaje. Comunicar, 19(37), 65-72. Recuperado el 8 de diciembre de 2015, de https://www.revistacomunicar.com/indice/articulo.php?numero=37-2011-08.

Padilla, S. (2008). Gestión de ambientes de aprendizaje constructivistas apoyados en la zona de desarrollo próximo. Apertura: Revista de Innovación Educativa, 6(5), 8-21. Recuperado el 8 de diciembre de 2015, de http://www.redalyc.org/ articulo.oa?id $=68800502$.

Parra, R., Castañeda, E., Delgadillo, M., Rueda, R., Turriago, O., \& Vargas, M. (1994). La Escuela Vacía. Bogotá, Colombia: Tercer mundo editores.

Rodríguez, A. (2013). ¿Cómo crear un ambiente de aprendizaje? Bogotá: Universidad de la Sabana. Recuperado el 10 de abril de 2017, de http://www.academia. edu/4536015/C\%C3\%B3mo_crear_un_ambiente_de_aprendizaje.

Santrock, J.W. (2014). Psicología de la educación. México D.F.: McGraw-Hill/ Interamericana Editores, S.A. DE C.V.

Soria, E. (2012). Habilidades emocionales, relacionales y afectivas eficaces en la estrategia docente. (Tesis de Maestría). Universidad de Valladolid, España. Recuperado el 5 de marzo de 2016, de https://uvadoc.uva.es/ bitstream/10324/2775/9/TFM-G\%2053.pdf.

Spranger, E. (1960). El Educador Nato. Buenos Aires, Argentina: Kapelusz S.A.

Uldemolíns, J. R., \& de la Rubia-Valladolid, I. P. (2003). Innovación educativa en Matemáticas. Comunicación y pedagogía: Nuevas tecnologías y recursos didácticos, (186), 8-14. Recuperado el 24 de marzo de 2016, de http://platea. pntic.mec.es/juldemol/pub/p9.pdf.

Vázquez, B., Jiménez, R., Mellado, V., \& Toboada, C. (2007). Un análisis de las interacciones en el aula. Recuperado el 5 de marzo de 2016, de http://www. eweb.unex.es/eweb/dcem/ar07investescuela.pdf. 